Fetal Diagnosis and Therapy
Fetal Diagn Ther 2018;43:156-160

DOI: $10.1159 / 000450606$
Received: May 2, 2016

Accepted after revision: September 5, 2016 Published online: October 6, 2016

\title{
Successful in utero Percutaneous Fetoscopic Release of a Wrapped Pleuro-Amniotic Shunt around the Fetal Arm: Case Report and Review of the Literature
}

\author{
Ahmed A. Nassr Rodrigo Ruano Jimmy Espinoza Alireza A. Shamshirsaz \\ Department of Obstetrics and Gynecology, Baylor College of Medicine, and Texas Children's Fetal Center, \\ Houston, Tex., USA
}

\section{Key Words}

Thoraco-amniotic shunt · Fetoscopy · Prenatal therapy

\begin{abstract}
Background and Objectives: Fetal pleural effusions are either primary or secondary to chromosomal or structural fetal abnormalities. In both cases, thoraco-amniotic shunts may be indicated to prevent life-threatening consequences. In this report, we describe a rare complication of shunts and successful in utero management of this complication. We also summarize the prior reports of shunt wrapping around fetal limbs and successful prenatal release of the shunts. Data Source: Search including PubMed, MEDLINE and EMBASE databases from inception till November 2015 was conducted for cases with shunt entrapment around fetal limbs with or without prenatal management. Results: Four cases including our case have been reported in the literature with wrapping of shunts around fetal limbs. Three of these cases were successfully managed prenatally using different interventional and anesthetic techniques. Conclusions: Prenatal shunts could be rarely complicated by wrapping of the shunt around fetal limbs with possible serious vascular and neurological consequences. Different techniques have been described for prenatal management of this condition. We describe a less invasive approach that could be done under local anesthesia in suitable cases.

(c) 2016 S. Karger AG, Basel
\end{abstract}

\section{KARGER}

(c) 2016 S. Karger AG, Basel

E-Mail karger@karger.com

www.karger.com/fdt

\section{Introduction}

Fetal pleural effusions could be primary in nature, which is mostly due to abnormal lymphatic drainage or delayed lymphatic development or secondary due to chromosomal or structural fetal abnormalities. In the latter group of fetuses, pleural effusions are frequently associated with fluid accumulation in other cavities and with fetal hydrops [1].

Although thoraco-amniotic shunts have been shown to improve perinatal survival in selected fetuses $[1,2]$, they are not free from complications [3]. Rare reported complications of thoraco-amniotic shunts include fetal chest wall deformities [4] and entrapment of the distal (amniotic) end of the shunt around a fetal limb [5-7]. Such entrapment could lead to vascular and/or neurological injury to the involved limb.

Recently, our group has reported on a two-port in$\mathrm{CO}_{2}$ fetoscopic procedure to release a thoraco-amniotic shunt wrapping around the fetal arm [5]. Our group recommended the use of $\mathrm{CO}_{2}$ as distention media when visualization of the fetal structures using the conventional percutaneous approach is anticipated to be very limited.

The objective of this report is to review the reported cases of shunt entrapment around the fetal limbs and to investigate the consequences and possible prenatal management of this rare complication. We also describe a case 
of successful in utero single-port percutaneous fetoscopic unwrapping of fetal thoraco-amniotic shunt. We discuss the surgical technical aspects related to this procedure.

\section{Methods}

We report a case of successful prenatal management of a rare complication of fetal shunt placement. Consent was obtained from the patient to report this case.

In addition, a search for articles that reported wrapping of prenatally placed shunts around fetal limbs was conducted. We did a comprehensive search including several databases (PubMed, MEDLINE and EMBASE) for relevant articles from inception till November 2015.

The following data was abstracted: type of intervention, type of shunt, fetal limb involved, technique of fetal intervention to release shunt, and type of anesthesia.

\section{Results}

\section{Case Presentation}

A 26-year-old G3P1 patient at 19 weeks and 6 days was referred to our fetal center because of bilateral pleural effusion that progressed later to hydrops (scalp edema, ascites and bilateral pleural effusion). Genetic amniocentesis demonstrated a normal karyotype. A fetal thoracocentesis was performed and pleural fluid was sent for cytological examination, which revealed predominately lymphocytes with some plasmacytoid forms and immature myeloid cells. Fetal echocardiogram revealed normal fetal cardiac structure and function.

At 20 weeks and 4 days, the patient elected to proceed with placement of bilateral fetal pleuro-amniotic shunts because of worsening fetal hydrops. A Harrison left pleural-amniotic shunt was successfully placed under ultrasound guidance. Due to fetal position, the decision was made to perform only a right fetal thoracocentesis to drain the pleural effusion using a 20-gauge needle. Hydrops resolved after the procedure; however, 2 weeks later, the shunt had migrated into the amniotic cavity and the fetus developed ascites with severe left pleural effusion and mild right effusion. Again, another Harrison left pleural-amniotic shunt was placed and right thoracocentesis was performed. This was followed by complete resolution of ascites and scalp edema. A few days later, the fetus developed hydrops again with severe right pleural effusion, accompanied by fetal echocardiographic findings suggestive of moderate tricuspid regurgitation. The decision was made to place a right thoraco-amniotic shunt. This time, we decided to place a Rocket shunt.
Placement of the Rocket shunt was done in the posterior fetal chest under the scapula. Placement was satisfactory with adequate visualization of both shunt ends in the correct place by ultrasound. Procedure was successful and hydrops resolved again. Weekly ultrasound revealed stable situation for 4 weeks. At 27 weeks, however, the right fetal arm was noticed to be wrapped by the distal end of the Rocket shunt without distal edema and with preserved blood flow as demonstrated by color Doppler (fig. 1-3). The decision was made not to intervene and continue with follow-up scans.

At 28 weeks, the left shunt had migrated again into the amniotic cavity and fetal hydrops reappeared with severe left pleural effusion and right mediastinal shift. A decision was made to place a left-sided Rocket pleural-amniotic shunt (primary objective) and to try to unwrap the shunt on the fetal right arm using the same uterine entry.

During the procedure, the left Rocket pleural-amniotic shunt was successfully placed without complications under maternal skin anesthesia and fetal intramuscular anesthesia. Before removing the Rocket trocar, we advanced a J wire through the Rocket trocar, removed the Rocked trocar and inserted a 12-Fr Cook disposable cannula into the amniotic cavity under ultrasound guidance. In addition, we infused a total $240 \mathrm{ml}$ of sterile Lactated Ringer fluid into the amniotic cavity. Subsequently, a 2.0$\mathrm{mm}$ 0-degree Hopkins scope (26008AA) was inserted into the amniotic cavity and the right fetal arm was visualized noticing two loops of the Rocket shunt wrapping around the upper part of the arm. Using the same port, a $2.0-\mathrm{mm}$ grasper was inserted into the $12-\mathrm{Fr}$ Cook cannula. With direct visualization by fetoscopy and indirect visualization by ultrasound, we successfully unwrapped the shunt from the right fetal arm, without needing to cut the device. Subsequent ultrasound examinations confirmed that the right arm was free from the shunt and the hydrops reversed completely.

At 33 weeks and 4 days, the patient had preterm premature rupture of membranes and underwent induction of labor at 34 weeks, which resulted in vaginal delivery of a male 2,085-gram baby with Apgar scores of 8 and 9 at 1 and 5 min, respectively. Fetal thoracic shunts were removed on the first day after birth and the neonate did not require the placement of chest drains. There was no evidence of skin indentation in the right arm. Postnatal vascular and neurological assessment revealed normal findings. At the time of this report, the 3-week-old baby is doing well (fig. 4). Based on clinical course of pleural effusion and the results of cytological examination, chylothorax has been suggested as etiological cause of pleural effusion in this case. 


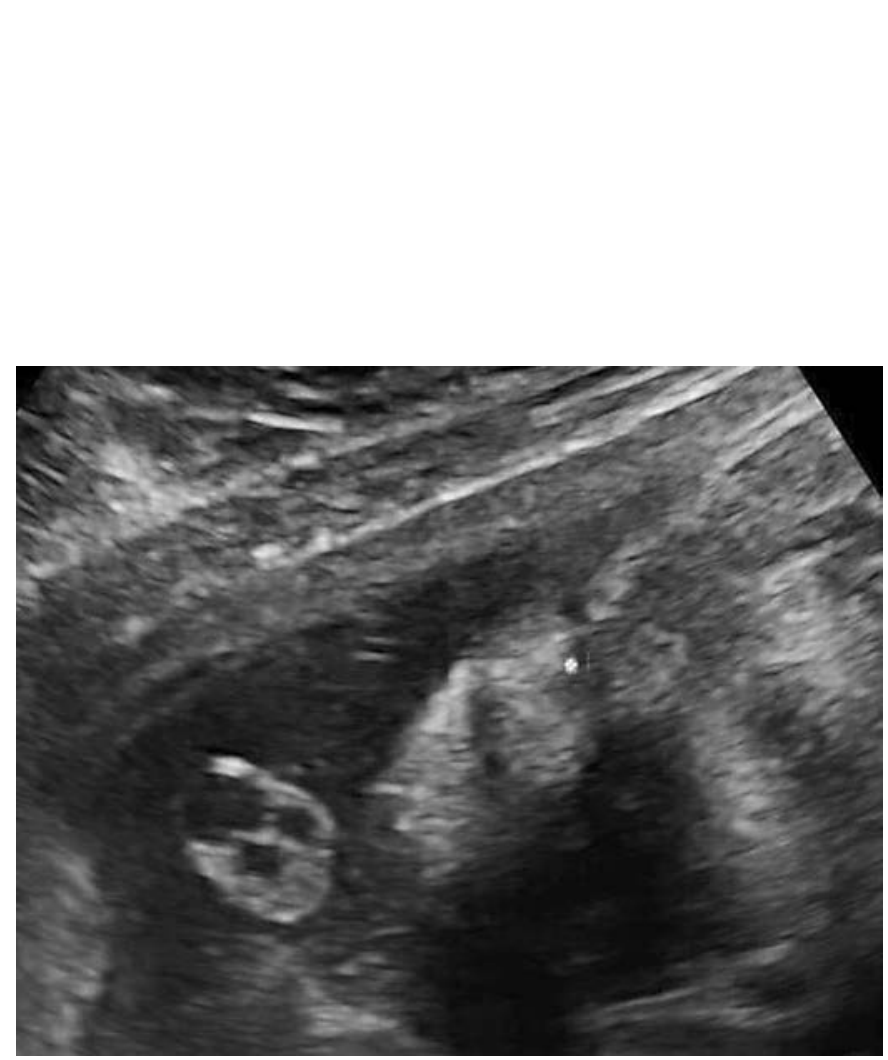

Fig. 1. Shunt wrapped around right fetal arm by two-dimensional ultrasound.

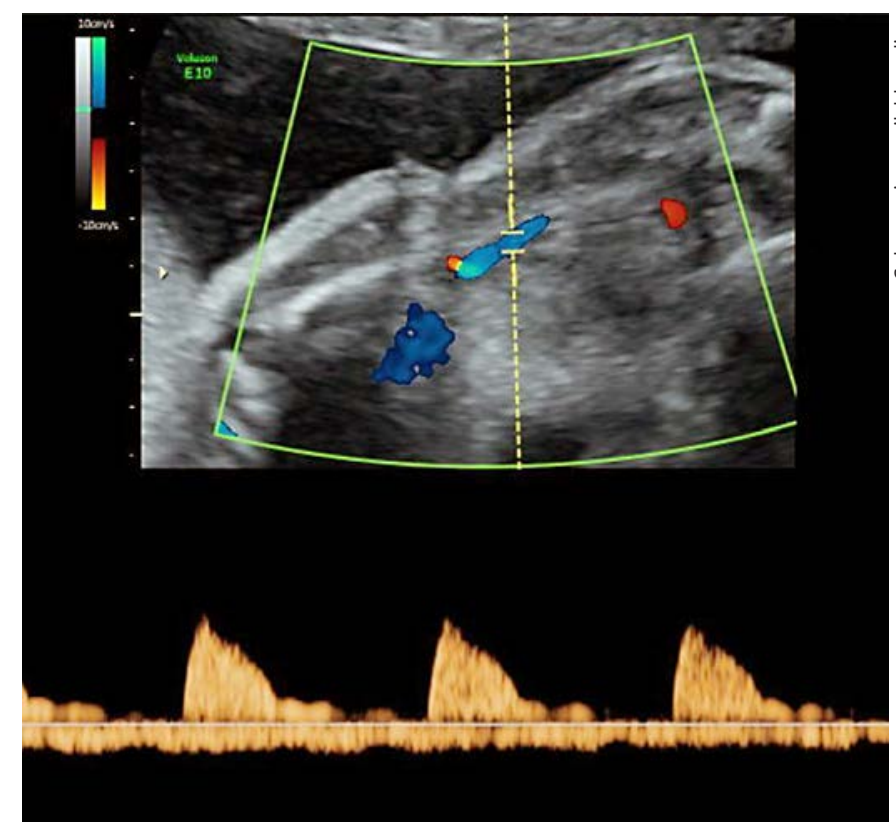

Fig. 3. Preserved blood flow in the arm by color Doppler.

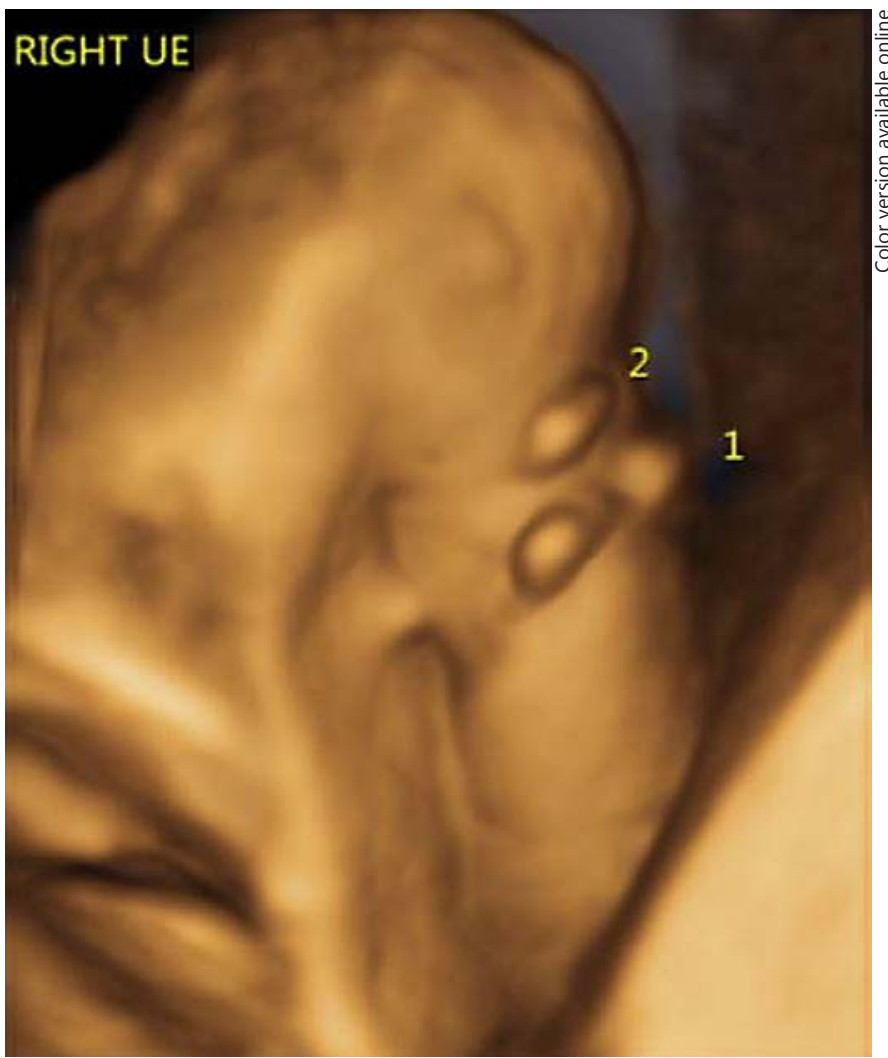

Fig. 2. Shunt wrapped around right fetal arm by three-dimensional ultrasound.

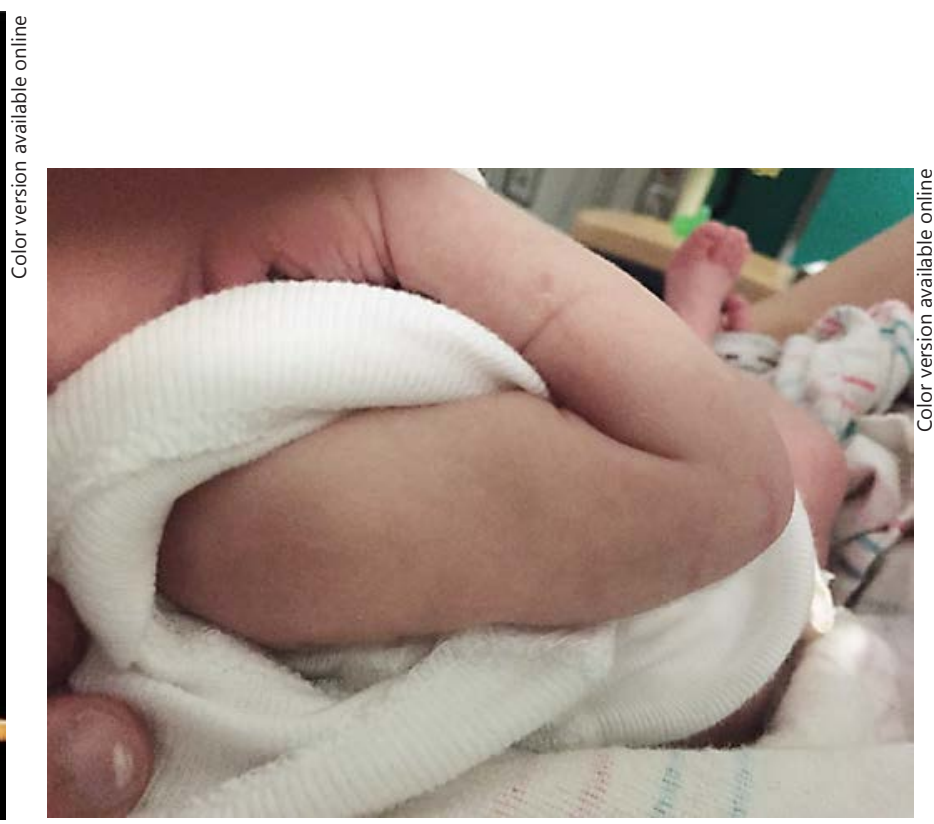

Fig. 4. Right arm postnatally. 
Table 1. Prior reports of shunt wrapping around fetal limbs and cases with successful prenatal release of shunts

\begin{tabular}{|c|c|c|c|c|c|c|c|}
\hline Author and year & $\begin{array}{l}\text { Type of } \\
\text { intervention }\end{array}$ & $\begin{array}{l}\text { Type of } \\
\text { shunt }\end{array}$ & $\begin{array}{l}\text { Fetal limb } \\
\text { involved }\end{array}$ & $\begin{array}{l}\text { Fetal intervention } \\
\text { to release shunt }\end{array}$ & $\begin{array}{l}\text { Site of insertion of } \\
\text { thoraco-amniotic } \\
\text { shunts }\end{array}$ & Anesthesia & Type of intervention \\
\hline Chan et al. [7], 2002 & $\begin{array}{l}\text { Vesico-amniotic } \\
\text { shunt }\end{array}$ & Rodeck & Left thigh & Yes & N/A & General & Fetoscopic release \\
\hline Recent case & $\begin{array}{l}\text { Pleuro-amniotic } \\
\text { shunt }\end{array}$ & Rocket & Right arm & Yes & $\begin{array}{l}\text { Right posterior part } \\
\text { of fetal chest below } \\
\text { the scapula }\end{array}$ & Local & Fetoscopic release \\
\hline
\end{tabular}

N/A = not applicable.

\section{Discussion}

We report a successful release of wrapped shunt on the fetal arm using a single-port percutaneous technique at the moment of a repeat pleural-amniotic shunt placement. In this case, our decision to use a Harrison shunt initially was related to early gestational age and small size of the fetus; then, after dislodgement of the previously placed Harrison shunt, we decided to use a Rocket shunt when the fetus was more advanced in gestation. A recent study published by our group to study factors associated with shunt dislodgment in patients with fetal lower urinary tract obstruction (LUTO) showed that type of shunt (Harrison versus Rocket) was the only prenatal factor associated with shunt dislodgment, and use of Rocket shunt in LUTO patients was associated with lower rates of dislodgment [8]. However, such association needs to be investigated in thoraco-amniotic shunts.

In the present case, we initially placed another shunt in the other side, and then we inserted a J wire in order to insert a 12-Fr Cook cannula. The decision of using a 12Fr cannula was made in advance with the objective of allowing the use of a fetoscope and a grasping forceps in a single-port access. This technique is less invasive when compared to other methods previously described and may be considered before performing the two-port under gas fetoscopy.

All thoracic shunts in our case were inserted in the posterior region of the chest below the scapula and great care was taken to ensure correct deployment of the thoracic and amniotic ends under close ultrasound guidance. It is not clear if a more posterior insertion site, which is not always technically feasible, could have avoided occurrence of this rare complication.

To our knowledge, this is the fourth reported case in the literature with this complication and the third successful shunt unwrapping procedure. Table 1 summarizes prior reports of shunt wrapping around fetal limbs and cases with successful prenatal release of shunts.

Although the first case reported by Brown and Nicolaides [6] did not mention any neonatal vascular or neurological consequences of shunt entrapment around the fetal arm despite conservative prenatal management, in the other two cases reported by Chan et al. [7] and Belfort et al. [5], there was a high prenatal suspicion of possible neurological and vascular complications as evidenced by abnormal Doppler flow studies [7], distal edema, and deep skin indentation in the involved limb [5].

In the latter two cases, release of shunt entrapment of the fetal limb was possible with favorable neonatal outcomes. In Chan et al. [7], case management was done through direct fetoscopic technique. The authors opted to divide the shunt with laparoscopic scissors at two points. This procedure was done under general anesthesia [7]. In the second case, the authors released the shunt from the arm despite the unfavorable location of the anteriorly placed placenta using laparotomy and two-port fetoscopy. The distention media used was $\mathrm{CO}_{2}$ to successfully complete the procedure [5].

In our case, we reported a successful unwinding of the pleuro-amniotic shunt from around the right fetal arm using single-port direct fetoscopy under local anesthesia through the same incision that was done for additional shunt placement. Although, the prenatal ultrasound eval- 
uations did not show ominous signs of impaired blood flow to the fetal arm or distal edema, there was a visible deep indentation and we decided to try unwinding the shunt at the same time of additional shunt placement to avoid possible complications that could potentially occur as the fetus grows.

Follow-up scans demonstrated gradual improvement in the skin indentation on the arm which was noticeable by ultrasound before delivery. After delivery, no skin indentation was observed in the right arm.

In conclusion, a less invasive single-port fetoscopic approach can be used under local anesthesia to release a shunt provided that the visualization during fetoscopy is anticipated to be adequate. If this approach proves to be technically difficult due to unfavorable placental position or inadequate visualization, then shifting to our previously described approach using laparotomy and two-port fetoscopy in a $\mathrm{CO}_{2}$-created space seems to be a reasonable alternative [5].

\section{Acknowledgement}

We would like to thank Dr. Michael Belfort, $\mathrm{MD}, \mathrm{PhD}$ for his valuable comments.

\section{Disclosure Statement}

The authors report no conflict of interests.

\section{References}

1 Yinon Y, Kelly E, Ryan G: Fetal pleural effusions. Best Pract Res Clin Obstet Gynaecol 2008;22:77-96.

2 Mallmann MR, Graham V, Rösing B, Gottschalk I, Müller A, Gembruch U, Geipel A, Berg C: Thoracoamniotic shunting for fetal hydrothorax: predictors of intrauterine course and postnatal outcome. Fetal Diagn Ther 2016, DOI: 10.1159/000446110.

3 Adams TM, Kunzier NB, Chavez MR, Vintzileos AM: Ultrasound-guided retrieval and position replacement of a dislodged fetal pleuro-amniotic shunt: a novel approach for a known complication of feto-amniotic shunting. Fetal Diagn Ther 2015;39:78-80.
4 Merchant AM, Peranteau W, Wilson RD, Johnson MP, Bebbington MW, Hedrick HL, Flake AW, Adzick NS: Postnatal chest wall deformities after fetal thoracoamniotic shunting for congenital cystic adenomatoid malformation. Fetal Diagn Ther 2007;22:435-439.

5 Belfort MA, Shamshirsaz AA, Whitehead WE, Ball R, Silver R, Ruano R, Espinoza J, Mann DG, McCullough L, Chervenak F: Unusual pleuro-amniotic shunt complication managed using a 2-port in- $\mathrm{CO}_{2}$ fetoscopic technique: technical and ethical considerations. Ultrasound Obstet Gynecol 2016;47: 123-124.
6 Brown R, Nicolaides K: Constriction band of the arm following insertion of a pleuro-amniotic shunt. Ultrasound Obstet Gynecol 2000; 15:439-440.

7 Chan FY, Borzi P, Cincotta R, Burke J, Tudehope $\mathrm{D}$ : Limb constriction as a complication of intra-uterine vesico-amniotic shunt: fetoscopic release. Fetal Diagn Ther 2002;17:315320.

8 Kurtz M, Koh CJ, Jamail GA, Sangi-Haghpeykar H, Shamshirsaz AA, Espinoza J, Cass DL, Olutoye OO, Olutoye OA, Braun MC, Roth DR, Belfort MA, Ruano R: Factors associated with fetal shunt dislodgement in lower urinary tract obstruction. Prenat Diagn 2016;36: $720-725$. 